\title{
Use of trifurcate arch graft in left ventricular assist device implantation via the sternal sparing approach
}

Prashant N. Mohite, MCh, Diana Garcia-Saez, MD, Aron-Frederik Popov, MD, and Andre R. Simon, MD, Harefield, United Kingdom

Current continuous flow left ventricular assist devices (LVADs) have become a routine therapeutic option for patients with end-stage heart failure and they can provide satisfactory long-term survival. ${ }^{1}$ Conventionally, LVAD implantation is performed through sternotomy with anastomosis of the outflow graft to the ascending aorta. Recently, several groups have demonstrated different approaches, like bilateral thoracotomy or thoracotomy with ministernotomy for LVAD implantation. ${ }^{2-4}$ We devised a novel technique wherein we utilize a trifurcate aortic graft (Vascutek Gelweave; Terumo, Renfrewshire, Scotland, United Kingdom) for an outflow graft that facilitates LVAD implantation via sternal-sparing bilateral thoracotomy.

\section{CLINICAL SUMMARY}

A 19-year-old man with dilated cardiomyopathy related to Becker muscular dystrophy who was receiving medical treatment deteriorated rapidly with commencement of liver and kidney dysfunction. Intravenous inotropic support was initiated. His echocardiography showed severely dilated

\footnotetext{
From the Department of Cardiothoracic Transplantation and Mechanical Support, Royal Brompton and Harefield National Health Service Trust, Harefield Hospital, Harefield, Middlesex, United Kingdom.

Disclosures: Authors have nothing to disclose with regard to commercial support.

Received for publication Nov 17, 2014; revisions received Jan 26, 2015; accepted for publication Jan 30, 2015; available ahead of print March 11, 2015.

Address for reprints: Prashant N. Mohite, MCh, Department of Cardiothoracic Transplantation and Mechanical Support, Harefield Hospital, Hill End Rd, Harefield,

Middlesex UB9 6JH, United Kingdom (E-mail: drprashantis@rediffmail.com).

J Thorac Cardiovasc Surg 2015;149:e113-4

$0022-5223 / \$ 36.00$

Copyright (c) 2015 by The American Association for Thoracic Surgery

http://dx.doi.org/10.1016/j.jtcvs.2015.01.057
}

ventricles with impaired systolic function (left ventricular ejection fraction of $26 \%$ ). Due to continuing deterioration, a long-term LVAD was implanted as a bridge to transplant.

The left ventricular apex was accessed through the left anterolateral thoracotomy via the fifth intercostal space followed by pericardiotomy and the ascending aorta was accessed through the right anterolateral thoracotomy via the fourth intercostal space. An HVAD ring (HeartWare Inc, Framingham, Mass) was sewn over the left ventricle just lateral to left anterior descending artery. A trifurcate aortic graft was prepared on back-table by closing the distal (ie, third) branch with 5-0 polypropylene suture and surgical adhesive (Figure 1). Four rings of the articulating strain relief were removed and the proximal end of the graft was fixed to the HVAD outflow. The graft was passed intrapericardially from left to right thoracotomy by the side of the right ventricle and right atrium. The graft was then passed under the superior vena cava according to techniques described elsewhere ${ }^{5}$ (Figure 2). The distal end of the main tube of the trifurcate graft was cut at an angle of $45^{\circ}$ and was anastomosed to the ascending aorta clamped with a Satinsky side-biting clamp. Heparin was given, the Satinsky clamp was removed, and the trifurcate graft was clamped just proximal to the anastomosis. The outflow tubing of the cardiopulmonary bypass (CPB) was connected to the middle (ie, second) branch of the trifurcate graft with the help of a two-thirds-inch connector. A clamp was applied in the graft between the first and second branches. Drainage was achieved via a cannula in the femoral vein and the patient was put on CPB. After coring out the left ventricle wall the HVAD pump was inserted into

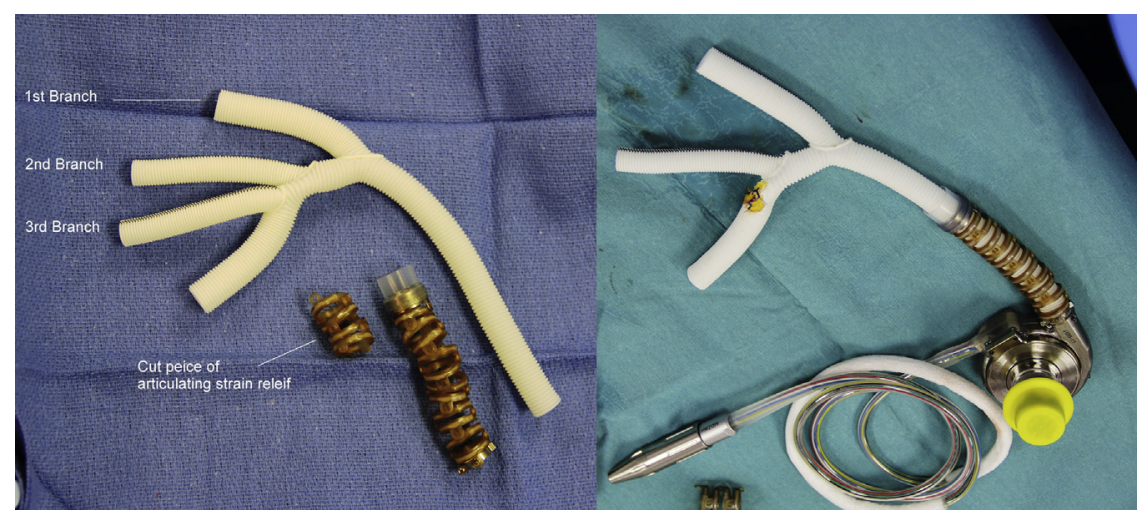

FIGURE 1. Trifurcate arch graft with HAVD (HeartWare Inc, Framingham, Mass). 


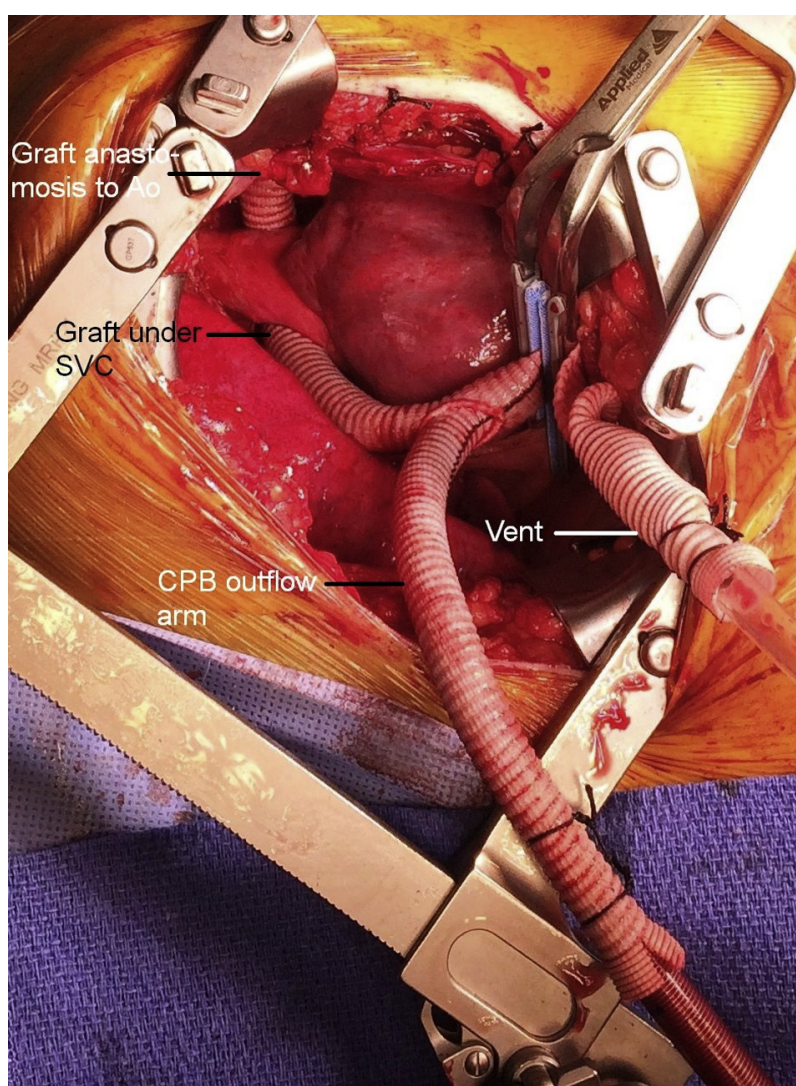

FIGURE 2. Trifurcate arch graft after anastomosis to the aorta $(A o)$ with cardiopulmonary artery bypass $(C P B)$ outflow and vent through its branches. $S V C$, Superior vena cava.

the ring. The pump and left ventricle were de-aired by running the pump with a vent into the trifurcate graft placed via the proximal (ie, first) branch. The patient was weaned off $\mathrm{CPB}$, remaining branches of the trifurcate graft were sewn off, and the patient's chest was closed. The patient made a fast recovery and was discharged home in 3 weeks.

\section{DISCUSSION}

The conventional sternotomy approach for LAVD implantation provides good exposure of the left ventricle and ascending aorta; however, the main drawback remains a difficult reentry at the time of LVAD explantation or exchange, aortic valve replacement, or heart transplantation with a potential risk of injuring the outflow graft. The bilateral thoracotomy approach-left subcostal for left ventricle apical inflow cannulation and right minithoracotomy for outflow graft anastomosis to ascending aorta-avoids sternotomy. ${ }^{2,3}$ However, with this technique access to the ascending aorta remains limited and becomes more intricate with $\mathrm{CPB}$ cannulae to the aorta and right atrium entering through the same incision. Although CPB may be achieved with peripheral cannulation via the femoral vessels, compromised flow to cerebral vessels and complications with groin wounds limits this approach.

We used a trifurcated arch graft in which 1 of the branches can be used at the CPB arterial arm, whereas another branch aids continuous venting and de-airing of the left ventricle. This not only provides more space to work through right minithoracotomy but also avoids additional perforations and maneuvers in the ascending aorta for CPB cannulation and vent. Venting and de-airing via a dedicated arm of trifurcated graft is continuous, efficient, and superior to needle graftotomy. Although trifurcate graft adds to the cost of the surgery, money is saved on aortic cannula, vent, and outflow graft. A custom-made graft with only 2 branches would save closure of the third branch of the commercially available trifurcate graft.

\section{CONCLUSIONS}

Use of a trifurcate arch graft as the outflow graft in LVAD implantation avoids ascending aortic cannulation and provides continuous venting and de-airing of the left ventricle. The technique facilitates LVAD implantation through sternal-sparing bilateral thoracotomy.

\section{References}

1. Takeda K, Takayama H, Kalesan B, Uriel N, Colombo PC, Jorde UP, et al. Long-term outcome of patients on continuous-flow left ventricular assist device support. J Thorac Cardiovasc Surg. 2014;148:1606-14.

2. Gregoric ID, La Francesca S, Myers T, Cohn W, Loyalka P, Kar B, et al. A less invasive approach to axial flow pump insertion. J Heart Lung Transplant. 2008; 27:423-6.

3. Schmitto JD, Molitoris U, Haverich A, Strueber M. Implantation of a centrifugal pump as a left ventricular assist device through a novel, minimized approach: upper hemisternotomy combined with anterolateral thoracotomy. J Thorac Cardiovasc Surg. 2012;143:511-3.

4. Popov AF, Hosseini MT, Zych B, Simon AR, Bahrami T. HeartWare left ventricular assist device implantation through bilateral anterior thoracotomy. Ann Thorac Surg. 2012;93:674-6.

5. Mohite PN, Popov AF, Mittal TK, Simon AR. Tunneling of ventricular assist device outflow graft rostral to superior vena cava. J Thorac Cardiovasc Surg. 2012;144:1519-20. 